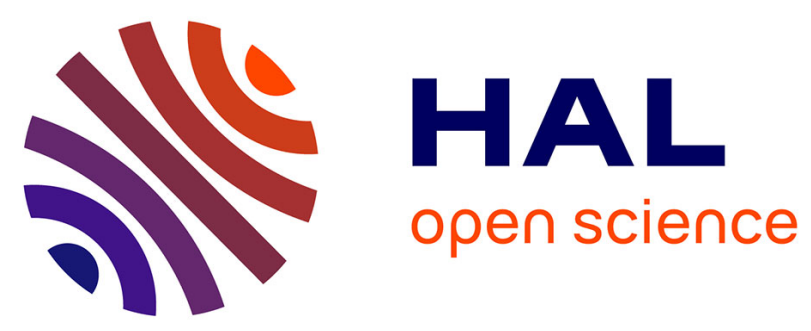

\title{
Reduction of PAPR of FBMC-OQAM systems by dispersive tone reservation technique
}

S. S. Krishna Chaitanya Bulusu, Hmaied Shaiek, Daniel Roviras

\section{To cite this version:}

S. S. Krishna Chaitanya Bulusu, Hmaied Shaiek, Daniel Roviras. Reduction of PAPR of FBMCOQAM systems by dispersive tone reservation technique. 2015 International Symposium on Wireless Communication Systems, Aug 2015, Brussels, France. pp.561-565, 10.1109/ISWCS.2015.7454408 . hal-02456361

\section{HAL Id: hal-02456361 https://hal-cnam.archives-ouvertes.fr/hal-02456361}

Submitted on 10 Mar 2020

HAL is a multi-disciplinary open access archive for the deposit and dissemination of scientific research documents, whether they are published or not. The documents may come from teaching and research institutions in France or abroad, or from public or private research centers.
L'archive ouverte pluridisciplinaire HAL, est destinée au dépôt et à la diffusion de documents scientifiques de niveau recherche, publiés ou non, émanant des établissements d'enseignement et de recherche français ou étrangers, des laboratoires publics ou privés. 


\title{
Reduction of PAPR of FBMC-OQAM Systems by Dispersive Tone Reservation Technique
}

\author{
S. S. Krishna Chaitanya BULUSU, Hmaied SHAIEK, and Daniel ROVIRAS \\ CEDRIC/LAETITIA, CNAM, Paris, France. \\ \{krishna-chaitanya.bulusu, hmaied.shaiek, daniel.roviras\}@cnam.fr
}

\begin{abstract}
Filter-bank multi-carrier (FBMC) with offsetquadrature amplitude modulation (OQAM) is being seriously considered for $5^{\text {th }}$ generation radio access technology. In this paper, we investigate the problem of peak-to-average power ratio (PAPR) reduction of FBMC-OQAM signals. We propose a novel scheme based on tone reservation (TR) scheme by considering the overlapping nature of FBMC-OQAM symbols. Any TR scheme uses the reserved tones to design a peak cancellation signal that reduces the PAPR. Simulation results indicate that the FBMC-OQAM with the proposed dispersive TR (DTR) scheme is not only efficient in reducing the PAPR but also closely lagging OFDM with classical TR scheme.
\end{abstract}

Keywords-Multicarrier communications, FBMC-OQAM, peak-toaverage power ratio, tone reservation, $5 G$.

\section{INTRODUCTION}

The Filter Bank Multi-Carrier (FBMC) based systems combined with Offset Quadrature Amplitude Modulation (OQAM) are gaining appeal as the front runner to become the radio waveform in forthcoming $5 \mathrm{G}$ Radio Access Technology (RAT) [1], [2], [3]. FBMC-OQAM has many notable features such as, excellent frequency localization, a power spectral density (PSD) with very low side lobes and robustness to frequency offsets. Armed with these properties, FBMC-OQAM seems to be more suitable candidate as the radio waveform for $5 \mathrm{G}$ RAT than OFDM, especially for asynchronous devices [1]. Like any other multi-carrier technique, FBMC-OQAM suffers from high peak-to-average power ratio (PAPR), degrading the efficiency of high power amplifier (HPA), which is an essential component in mobile devices and is inherently non-linear. The conjunction between high PAPR and nonlinear HPA hampers badly the frequency localization property due to possible spectral regrowth. So, there is a dire need to probe for novel methods relevant to PAPR reduction and HPA linearization. In this paper, we will investigate PAPR reduction methods for FBMC-OQAM systems.

Numerous PAPR reduction methods for OFDM do exist. The tone reservation (TR) technique is an adding signal method [4]. It is very simple, agile and it does not cause any interference to the original data signal. The classical PAPR reduction schemes, proposed for OFDM, cannot be directly applied to FBMC-OQAM, as the later have overlapping signal structure. In [5], the authors proposed sliding window tone reservation technique for FBMC-OQAM signals, based on projection onto convex sets (POCS) method proposed for OFDM. POCS has less computational complexity; albeit, it is a sub-optimal approach. Optimal result can be obtained, when we pose the optimization problem in TR, as quadratically 978-1-4673-6540-6/15/\$31.00 (c)2015 IEEE constrained quadratic program (QCQP) and the same is used in this paper. We believe that in the existing literature, no optimal PAPR reduction scheme based on tone reservation has been suggested yet for FBMC-OQAM systems. In this paper, we propose a dispersive tone reservation (DTR) for reducing the PAPR of FBMC-OQAM signals, by taking into account the dispersive nature of FBMC signal structure. The position of peak reduction tones (PRTs) make significant impact on the PAPR reduction performance and, it has been shown that the random PRTs yield very good results than equidistant or contiguous PRTs [4]. In this paper, we have proposed the determination of PRT positions based on the kernel method that has been adapted to the particular case of FBMC-OQAM systems.

Rest of the paper is organized as follows: Section II presents an overview of the FBMC-OQAM signal structure and the PAPR analysis. In Section III, the classical TR scheme is presented along with its extension to DTR scheme, in order to have a better comprehension about incapability of classical TR for a FBMC-OQAM system. In Section IV, the simulation results are presented and the conclusion of the paper is given in Section V.

\section{PRESENTATION OF FBMC-OQAM SYSTEM AND PAPR ANALYSIS}

\section{A. Overview of FBMC-OQAM signal structure}

Let us assume, we need to transmit $M \times N$ complex input symbols in a FBMC-OQAM system over $N$ tones. We denote the $m^{\text {th }}$ symbol over $n^{\text {th }}$ sub-carrier as $X_{m, n}$. In a FBMCOQAM system, we transmit real symbols at interval $\frac{T}{2}$ [3], where, $T$ is the time period of a FBMC-OQAM symbol. The $m^{\text {th }}$ input symbol vector, which is a collection of $N$ complex input symbols can be written as

$$
\mathbf{X}_{m}=\left(X_{m, 0}, X_{m, 1}, \ldots, X_{m, N-1}\right)^{\top}, 0 \leq m \leq M-1
$$

where, $(.)^{\top}$ is the transpose operator. The OQAM mapping of the complex input symbol vectors $\left\{\mathbf{X}_{m}\right\}_{m=0}^{M-1}$ into real symbol $\left\{a_{m^{\prime}, n}\right\}_{m^{\prime}=0}^{2 M-1}$ is done as shown below

$$
\begin{aligned}
m^{\prime} & = \begin{cases}2 m, & \mathrm{~m}^{\prime} \text { is even } \\
2 m+1, & \mathrm{~m}^{\prime} \text { is odd }\end{cases} \\
a_{m^{\prime}, n} & =\left\{\begin{array}{l}
(1-\beta) \cdot R_{m}^{n}+\beta \cdot I_{m}^{n}, \quad \mathrm{~m}^{\prime} \text { is even } \\
\beta \cdot R_{m}^{n}+(1-\beta) \cdot I_{m}^{n}, \quad \mathrm{~m}^{\prime} \text { is odd }
\end{array}\right. \\
\beta & =n \text { modulo } 2,0 \leq n \leq N-1
\end{aligned}
$$

Where, $R_{m}^{n}$ and $I_{m}^{n}$ are the real and imaginary parts of the complex elements in $\left\{\mathbf{X}_{m}\right\}_{m=0}^{M-1}$. In OQAM, a time staggering 
of $T / 2$ will be done either on the real or on the imaginary parts of complex symbols in an input symbol vector according to the sub-carrier index. This rule of time-staggering has mathematical significance, which can be seen in [7]. After this OQAM mapping, the real symbols undergo poly-phase filtering that involves IFFT along with filtering by a synthesis filter bank of a prototype filter and the obtained continuoustime base-band FBMC-OQAM signal $s(t)$ is given by [8]

$$
\begin{aligned}
s(t) & =\mathcal{G}\left\{\left\{\mathbf{X}_{m}\right\}_{m=0}^{M-1}\right\} \\
& =\mathcal{G}\left\{\mathbf{X}_{0}, \mathbf{X}_{1}, \ldots, \mathbf{X}_{M-1}\right\} \\
& =\sum_{m^{\prime}=0}^{2 M-1} \sum_{n=0}^{N-1} a_{m^{\prime}, n} h\left(t-m^{\prime} T / 2\right) e^{j \frac{2 \pi}{T} n t} e^{j \varphi_{m^{\prime}, n}}
\end{aligned}
$$

where,

- $\mathcal{G}\{$.$\} is the FBMC-OQAM modulation function,$

- $a_{m^{\prime}, n}$ are OQAM mapped from $\mathbf{X}_{m}$,

- $h(t)$ is the prototype filter impulse response,

- $\varphi_{m^{\prime}, n}$ is the phase term, equals to $\frac{\pi}{2}\left(m^{\prime}+n\right)-\pi m^{\prime} n$.

Let $s_{m}(t)$ be the $m^{t h}$ FBMC-OQAM symbol that is obtained after the modulation of the corresponding $m^{\text {th }}$ input symbol vector $\mathbf{X}_{m}$. Then, the continuous time domain signal for all the $M$ input signal vectors at the FBMC-OQAM transmitter output can be written as

$$
s(t)=\sum_{m=0}^{M-1} s_{m}(t-m T), 0 \leq t<\infty
$$

In this paper, we use PHYDYAS filter as prototype filter, $h(t)$ that was designed based on frequency sampling technique [2].

\section{B. Overlapping nature of FBMC-OQAM signals}

The duration of $h(t)$ impacts the FBMC-OQAM signal, causing adjacent FBMC-OQAM symbols to overlap. In PHYDYAS filter, most of the energy lies within the main lobe. In order to elucidate the overlapping nature, one need to get to know about the mean power profile of a FBMC-OQAM signal, which is defined as

$$
P_{a v g}[s(t)]=\mathbb{E}\left[|s(t)|^{2}\right]
$$

where, $\mathbb{E}[$.$] is the expectation operator. Due to time-dispersive$ nature, the real and imaginary parts of the symbol have an offset of $\frac{T}{2}$ and both parts have a span of $4 T$, which is directly linked to the length of $h(t)$. It is evident from Fig. 1 that the power profile of the FBMC-OQAM symbols spans over 4.5T, where as in the case of OFDM, it spans over $T$. Unlike OFDM, we can also observe that most of the energy of a FBMCOQAM symbol lies in its succeeding two symbols rather than its own period interval.

\section{PAPR analysis}

The PAPR is a random variable that is an appurtenant parameter in measuring the sensitivity of a non-linear HPA; when a non-constant envelop input need to be transmitted. Every multi-carrier modulation system have high PAPR, which poses a severe challenge to the RF design of HPAs. This prompts to seriously probe for PAPR reduction techniques. The

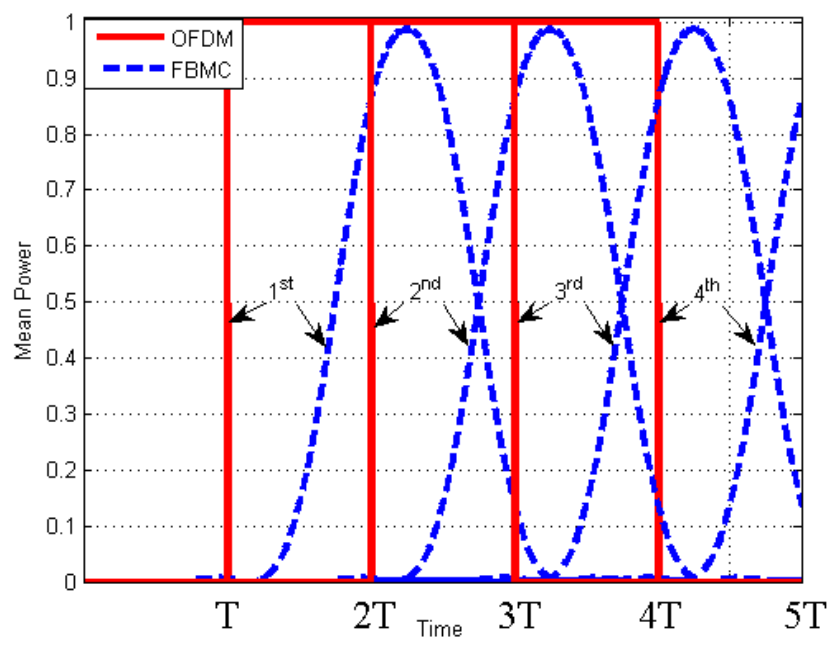

Fig. 1. Illustration of mean power profile of OFDM and FBMC-OQAM symbols.

PAPR of the continuous-time base-band signal $s(t)$ transmitted during a symbol period $T$ is defined by,

$$
\operatorname{PAPR}_{s(t)}=\frac{\|s(t)\|_{\infty}^{2}}{\frac{1}{T} \int_{0}^{T}\|s(t)\|^{2} . d t}
$$

where, $\|\cdot\|$ and $\|\cdot\|_{\infty}$ denote Euclidean and infinity norm, respectively. The complementary cumulative density function (CCDF) of PAPR is a useful parameter to analyze the PAPR, which is defined as the probability that the PAPR of the discrete-time signal exceeds beyond a given threshold that is denoted by $\gamma$ and thereby it can be evaluated as $\operatorname{Pr}\left\{P A P R_{s(t)} \geq \gamma\right\}$.

\section{PAPR REDUCTION USING DISPERSIVE TR}

\section{A. Classical TR for OFDM}

Classical TR was first introduced in [4]. The idea behind TR is to isolate energy used to cancel large peaks to a predefined set of tones, namely peak reserved tones (PRTs). These tones do not carry any useful information and are orthogonal to the data tones (DTs). This orthogonality makes recovering the data trivial. Stated mathematically, the resulting signal to be transmitted will be

$$
s(t)=d(t)+c(t), 0 \leq t<\infty
$$

where, $c(t)$ is the peak cancellation signal and $d(t)$ is the data signal (i.e. related to data only). $s(t)$ can be represented in frequency domain as $X[n]$, given by

$$
X[n]= \begin{cases}D[n], & n \in \mathcal{B}^{c} \\ C[n], & n \in \mathcal{B}\end{cases}
$$

where,

- $\mathcal{B}$ is the set of locations of PRTs, which is of length $R$,

- $D[n]$ is DT set and $D[n]=0$, for $n \in \mathcal{B}$,

- $C[n]$ is PRT set and $C[n]=0$, for $n \in \mathcal{B}^{\mathrm{c}}$. 
The aim of TR scheme is to compute the optimal values of reserved tones subject to

$$
\begin{aligned}
& \hat{c}(t)=\underset{c(t)}{\arg \min } P A P R_{s(t)} \\
& \equiv \underset{C[n]}{\arg \min }\left[\| \mathcal{B}\left[d(t)+\mathcal{F}^{-1}\{C[n]\} \|_{\infty}^{2}\right], 0 \leq t<\infty\right.
\end{aligned}
$$

where, $\mathcal{F}^{-1}($.$) is the inverse discrete Fourier transform. The$ aim is to select proper PRT set, so that the PAPR of the signal is reduced. The optimal $\hat{c}(t)$ can be obtained by solving (11), which can be done by convex optimization algorithms such as QCQP [4], POCS [9], gradient search [4], etc. The QCQP has the computational complexity of $\mathcal{O}\left(R N^{2}\right)$ and yields to the optimal result. The sub-optimal approaches such as POCS and gradient search has the computational complexity of $\mathcal{O}(N \log N)$ and $\mathcal{O}(N)$ respectively. In classical TR, the reserved tones can be removed easily at the receiver side.

1) Determination of $\mathcal{B}$ for OFDM: Nevertheless, finding the optimal PRT location set, $\mathcal{B}$ is a hard problem and it heavily impacts the performance of any TR scheme. The peak reduction kernel, $\mathcal{K}$ is designed as per the method suggested in [6], where, minimization of kernel side-lobes is the design criteria for choosing the optimal random PRT locations. Once $\mathcal{B}$ with a given size $\mathrm{R}$, is determined, the same can be used in solving (11).

\section{B. Dispersive $T R$}

OFDM signals do not have overlapping nature and thus classical TR scheme is sufficient since, $c(t)$ is determined independently for each symbol. Due to the overlapping nature of FBMC-OQAM symbols, direct implementation of classical TR scheme yields poor PAPR reduction performance.

In this section, we propose a novel scheme, namely, dispersive TR (DTR) to reduce the PAPR of the FBMC-OQAM systems. The aim of the DTR is to consider the overlap of the past symbols on the current $m^{\text {th }}$ symbol, when solving the optimization problem. The proposed scheme deals with the time dispersive nature of the FBMC-OQAM signals and hence termed as DTR. The prototype filter considered in our analysis is PHYDYAS one. It has to be noted that the peak cancellation signals of the preceding overlapping symbols are fixed, while the current symbol's peak cancellation signal $\hat{c}_{m}(t)$ is obtained by solving the optimization problem.

1) Determination of $\mathcal{B}$ for FBMC-OQAM: The determination of PRT locations for FBMC-OQAM is different than that of OFDM as the kernel cannot obey the circular property, due to the presence of prototype filter. The peak reduction kernel, $\mathcal{K}$ should be Dirac-type, i.e. as close as possible to the unit impulse symbol $\boldsymbol{\delta}$ of length $4.5 T$, which is defined as

$$
\boldsymbol{\delta}\left(t-t_{0}\right)= \begin{cases}1, & t=t_{0}, 0 \leq t<4.5 T \\ 0, & t \neq t_{0}, 0 \leq t<4.5 T\end{cases}
$$

where, $t_{0} \in[0,4.5 T)$. In the case of OFDM, $\mathcal{K}$ obeys circular property and hence $t_{0}$, (the maximum of the Dirac-type kernel) can be chosen to be 0. By multiplying the PRT tones by an exponential will change the positions of $t_{0}$. In FBMC-OQAM, it is totally different. A circular shift of the kernel maximum position is not possible by a simple multiplication of the PRTs. This is due to the presence of the prototype filter. Looking at Fig. 1, it is clear that the maximum mean energy of the first FBMC-OQAM symbol is located at $2.25 T$. In fact, the maximum of $h(t)$ is at $t=2 T$ and the delay of the imaginary part (due to OQAM modulation), gives finally a maximum in $t=2.25 T$. So, we will choose $t_{0}=2.25 T$ in order to generate the kernel so that, the unit impulse symbol $\delta$ defined in (12) should have its peak corresponding to the maximum mean power of the FBMC-OQAM symbol. This choice of $t_{0}=$ $4.5 T$ guarantees that we will have the minimal power on the reserved tones in order to get a Dirac-type kernel. Choosing $t_{0}=T$, for example, should result in a higher power on the PRTs for having the same maximum for the kernel. For FBMCOQAM, design of $\mathcal{K}$ is subject to

$$
\mathcal{K}=\underset{\mathcal{B}, \mathcal{K}_{0}}{\arg \min }\left[\left\|\mathcal{G}\left(\mathcal{K}_{0}\right)-\boldsymbol{\delta}\left(t-t_{0}\right)\right\|^{2}\right]
$$

where $\mathcal{K}_{0}$ is any peak reduction kernel that can be generated based on a given $\mathcal{B}$, which obeys the conditions $\mathcal{K}_{0}[n] \neq 0$, for $n \in \mathcal{B}$ and $\mathcal{K}_{0}[n]=0$, for $n \in \mathcal{B}^{c}$. For $R$ reserved tones out of $N$ tones, there will be $\left(\begin{array}{l}N \\ R\end{array}\right)$ possible combinations in total. For every combination of PRT locations, we compute a kernel. Out of all computed kernels, we pick the one, which has the least secondary peaks, i.e. the difference between the central peak and the secondary ones will be maximum. This kernel will not be used as it is in the following; but, only the PRT locations of it will be used deeming them as optimal PRT locations.

\section{2) Algorithm: The DTR algorithm is given below}

Step 1: Firstly, we compute the PRT location set $\mathcal{B}$, based on the peak reduction kernel $\mathcal{K}$, obtained from (13). We generate $M$ complex DT sets $\left\{\mathbf{D}_{m}\right\}_{m=0}^{M-1}$ as per (1) and by putting zeros in the PRT locations, for $0 \leq n \leq N-1$, as shown below

$$
\mathbf{D}_{m}[n]= \begin{cases}\text { data }, & n \in \mathcal{B}^{c} \\ 0, & n \in \mathcal{B}\end{cases}
$$

Then, We intialize $m=1$. (We should know that, $\mathbf{X}_{m}=\mathbf{D}_{m}+\mathbf{C}_{m}$ and our aim is to compute the optimal PRT set, $\mathbf{C}_{m}$, by solving the optimization problem.)

Step 2: The current FBMC-OQAM symbol $\tilde{s}_{m}(t)$, is obtained by FBMC-OQAM modulation for the $m^{t h}$ DT set $\mathbf{D}_{m}$ as per (5) and the signal generated by past symbols, denoted by $\dot{o}_{m}(t)$, is added to the current symbol as below

$$
\begin{aligned}
\dot{s}_{m}(t) & =\mathcal{G}\left\{\mathbf{X}_{0}, \mathbf{X}_{1}, \ldots, \mathbf{X}_{m-1}, \mathbf{D}_{m}\right\} \\
& =\underbrace{\sum_{m^{\prime}=0}^{2 m-1} \sum_{n=0}^{N-1} \dot{b}_{m^{\prime}, n} h\left(t-m^{\prime} T / 2\right) e^{j \frac{2 \pi}{T} n t} e^{j \varphi_{m^{\prime}, n}}}_{\text {overlapping past symbols }} \\
& +\underbrace{\sum_{m^{\prime}=2 m}^{2 m+1} \sum_{n=0}^{N-1} \dot{a}_{m^{\prime}, n} h\left(t-m^{\prime} T / 2\right) e^{j \frac{2 \pi}{T} n t} e^{j \varphi_{m^{\prime}, n}}}_{\text {current symbol }} \\
& =o_{m}(t)+\tilde{s}_{m}(t)
\end{aligned}
$$

where,

- $\dot{s}_{m}(t) \neq 0$ from $t=\left[0,\left(m+\frac{1}{2}\right) T+4 T\right)$,

- $\dot{a}_{m^{\prime}, n}$ are OQAM mapped from $\mathbf{D}_{m}$,

- $\dot{b}_{m^{\prime}, n}$ are OQAM mapped from $\mathbf{X}_{0}, \mathbf{X}_{1}, \ldots, \mathbf{X}_{m-1}$. 


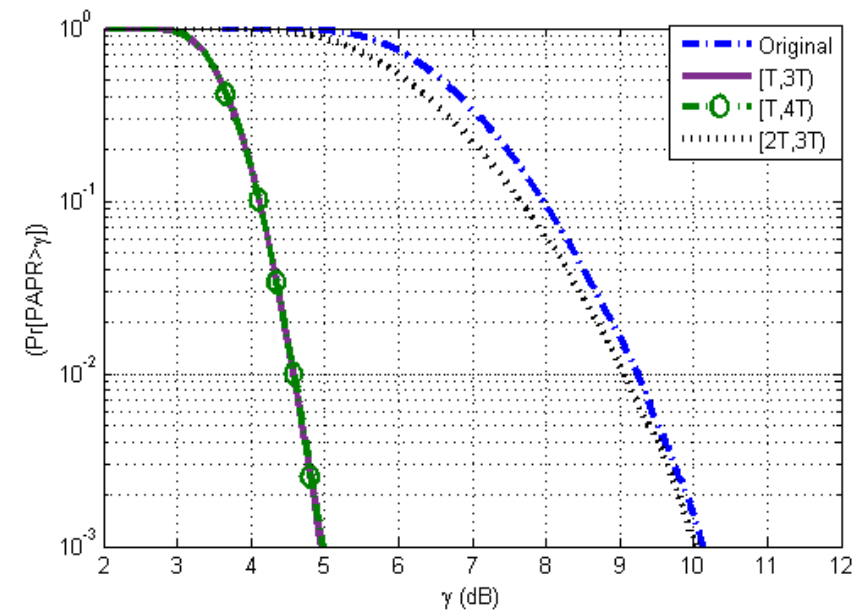

Fig. 2. CCDF of PAPR plot for FBMC-OQAM symbols with PAPR calculated over $[T, 3 T),[T, 4 T)$ and $[2 T, 3 T)$ with 8 PRTs (i.e. R=8).

Step 3: The peak cancellation signal of $\dot{s}_{m}(t)$, denoted by $\hat{c}_{m}(t)$ is obtained by convex optimization by solving the below optimization problem as QCQP, on a certain interval $T_{0}=$ $\left[m T+T_{a}, m T+T_{b}\right)$, which is any arbitrary interval within the $[m T, m T+4.5 T)$ interval

$$
\hat{c}_{m}(t)=\underset{C_{m}}{\arg \min }\left[\left\|\dot{s}(t)+\mathcal{G}\left\{\mathbf{C}_{m}\right\}\right\|_{\infty}^{2}\right], m T+T_{a} \leq t<m T+T_{b}
$$

where, $t \in T_{0}, T_{a} \geq 0$ and $T_{b}<4.5 T$.

Step 4: Once $\hat{c}_{m}(t)$ is obtained, then construct the optimal current input symbol vector

$$
\mathbf{X}_{m}=\mathbf{D}_{m}+\mathbf{C}_{m}
$$

Step 5: Increment $m$ by 1 and go to Step 2, until $m=M-1$.

\section{Simulation Results}

Simulations are done for a FBMC-OQAM signal that has been generated from $10^{6}$ 4-QAM symbols with 64 tones. The PHYDYAS prototype filter was used, which spans for $4 T$ [2]. CCDF has been considered as the measurement of PAPR reduction performance. In the simulation figures, the curve "Original" represents the performance of the FBMC-OQAM system without TR (i.e. $\mathcal{B}$ is a null set). Where as, the "DTR" and "TR" represents the dispersive and classical TR schemes respectively. The classical TR is done by substituting the (17) with $\dot{o}_{m}(t)=0$.

\section{A. Impact of variation of $T_{o}$ duration}

When computing the PAPR, the interval $\left[T_{a}, T_{b}\right)$ of duration $T_{o}$ seems to make a significant impact in the performance of DTR scheme as depicted in Fig. 2. By varying the duration over which the PAPR is calculated, we can see how it is affecting the efficiency of the DTR scheme. Looking at Fig. 1, it is evident that the interval $T_{0}$ goes from $T_{a}=0$ to $T_{b}=4.5 T$. Nevertheless, because of the power profile of the prototype filter, the interval $T_{0}$ can be reduced. Reducing the interval $T_{0}$ reduces the complexity of the optimization problem.

Choosing $T_{a}=T$ and $T_{b}=3 T$ or $4 T$ give the same performance because, for $t \leq T$, there is no significant

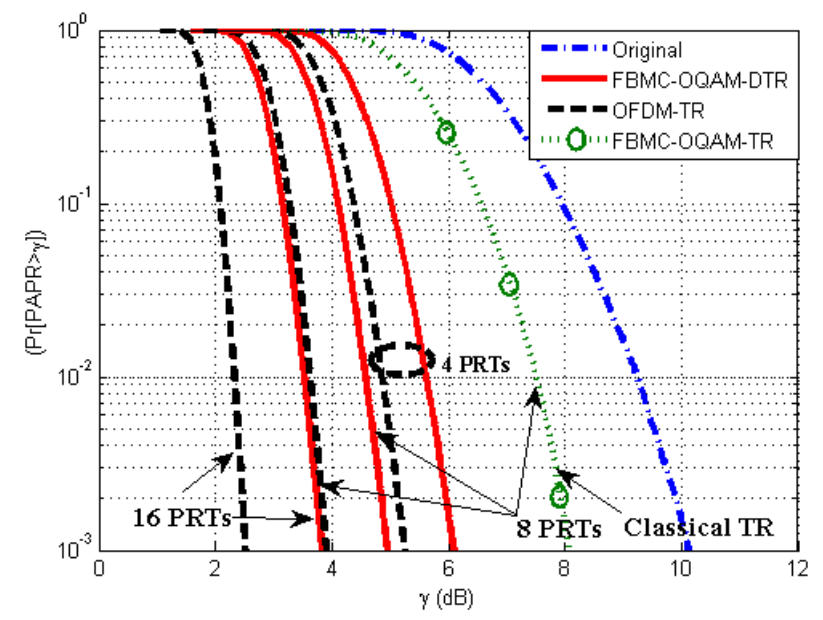

Fig. 3. CCDF of PAPR plot for FBMC-OQAM symbols with PAPR calculated over $[T, 3 T)$ with size of PRT set, $R=\{4,8,16\}$.

contribution of $s_{m}(t)$ (see Fig. 1) and for $t \geq 3 T$, it is the same. If we choose $T_{a}=2 T$ and $T_{b}=3 T$, there is a significant degradation on the performance, because a significant part of the energy of $s_{m}(t)$, lies between $T$ and $2 T$. In conclusion, it is sufficient to make the optimization problem of (18), for an interval $T_{0}$ going from $T_{a}=T$ to $T_{b}=3 T$.

\section{B. Impact of size of $\mathcal{B}$}

In any TR scheme, the size of $\mathcal{B}, R$ impacts the performance of the PAPR reduction [6]. To analyze the impact of $R$, we have considered different sizes of $R=\{4,8,16\}$ and fixed $T_{o}=[T, 3 T)$. The values at $10^{-3}$ of CCDF of PAPR in the Fig. 3, has been summarized in Table I.

TABLE I. CCDF OF PAPR AT $10^{-3}$ VALUE (IN $d B$ )

\begin{tabular}{|c|c|c|c|c|}
\hline MODULATION TYPE & REDUCTION SCHEME & $R=4$ & $R=8$ & $R=16$ \\
\hline OFDM & Classical TR & 5.3 & 3.9 & 2.5 \\
\hline FBMC-OQAM & Dispersive TR & 6.1 & 4.9 & 3.8 \\
\hline FBMC-OQAM & Classical TR & - & 8.1 & - \\
\hline
\end{tabular}

The exploitation of the overlapping nature of the FBMCOQAM signals can significantly impact the PAPR reduction. From Table I, it is clear that the PAPR reduction performance of FBMC-OQAM with classical TR is outperformed by the one with DTR scheme by $3.2 d B$, for $R=8$.

Also in the same figure, we can notice that the CCDF of PAPR for FBMC-OQAM with DTR is closely trailing that of OFDM with classical TR by roughly $1 d B$, for $R=\{4,8,16\}$, respectively. Another important observation is that, as size of $\mathcal{B}$ is getting increased; we can see the trail gap between CCDF curves of OFDM and FBMC-OQAM is getting increased. The reason for performance lag of DTR scheme is that there is a possibility that PAPR reduction done for the current symbol can be hampered by the future symbols, which is an inherent liability for schemes that opt for symbol-by-symbol approach. The probability of such occurrences increases along with the number of PRTs and this explains the increase in performance lag of FBMC-OQAM with DTR w.r.t. the OFDM with classical TR. 


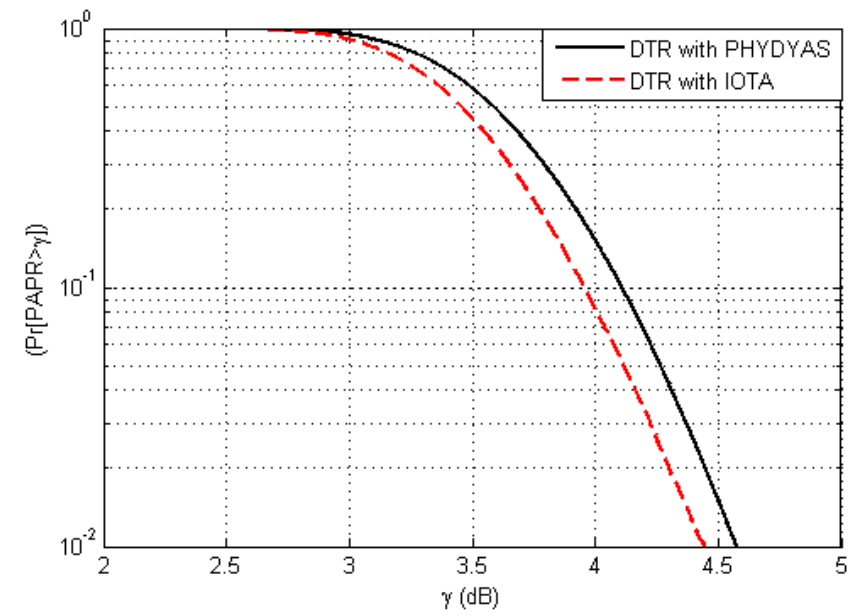

Fig. 4. CCDF of PAPR plot for FBMC-OQAM symbols with PAPR calculated over $[T, 3 T)$ with size of PRT set, $R=8$.

\section{Impact of variation in prototype filter}

In order to understand the impact of variation in prototype filter over the PAPR reduction performance DTR scheme, simulation has been done by considering isotropic orthogonal transform algorithm (IOTA) filter [10] as the prototype filter. IOTA prototype function is a special case of extended Gaussian function (EGF). We can notice from Fig. 4, that the DTR scheme fares marginally better in a FBMC-OQAM system with IOTA filter than PHYDYAS one. This is due to the fact that the signal energy spread is lower in the case of IOTA filter leading to less symbol overlapping than the PHYDYAS one.

\section{Impact of PRT locations}

Simulation results show that the location of PRTs have significant impact on the PAPR reduction of the FBMC-OQAM systems with DTR scheme. In the legend of Fig. 5, "equidistant" and "random (kernel)" indicate the PRT locations that are uniformly spaced, and obtained based on kernel method, respectively. Whereas "random 1","random 2" and "random 3" are three different randomly chosen (without based on kernel method) PRT locations. From Fig. 5, it can be inferred that the equi-distant case always yields non-optimal performance and it is better to opt for random PRT locations obtained by kernel method; as the PAPR reduction performance of the DTR scheme based on the later depends on luck rather than reason.

\section{CONCLUSION}

FBMC-OQAM systems has different signal structure than OFDM systems. So, a TR based scheme, namely, DTR has been proposed for FBMC-OQAM systems. Simulation results show that, although we consider the overlap of the past symbols, the duration of signal considered for optimization has significant impact on the PAPR reduction performance and the proposed DTR scheme is quite effective in reducing the PAPR of the FBMC-OQAM signal and the PRT locations also play vital role in that aspect. Albeit, this scheme is closely lagging behind the OFDM with classical TR in terms of PAPR reduction performance.

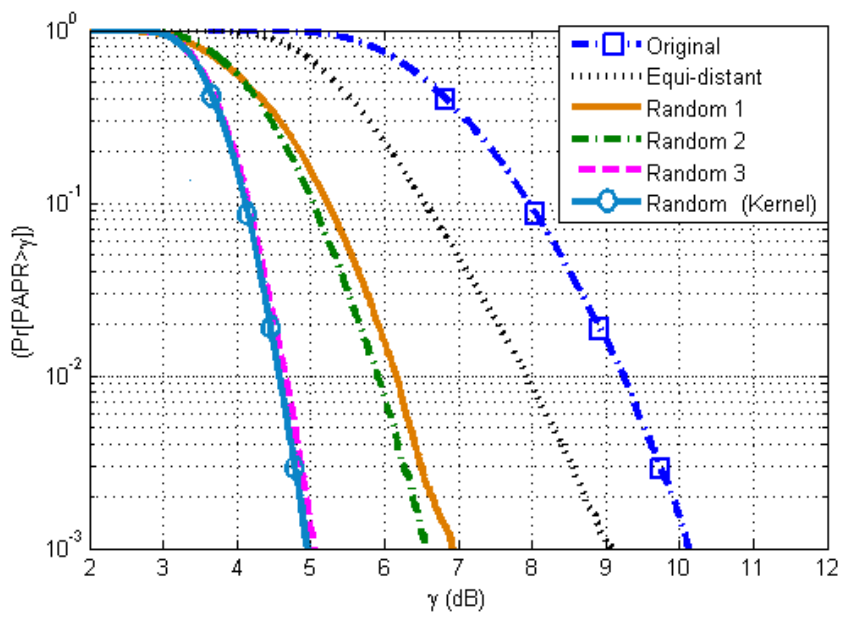

Fig. 5. CCDF of PAPR plot for FBMC-OQAM symbols with PAPR calculated over $[T, 3 T)$ with 8 PRTs (i.e. $\mathrm{R}=8$ ) for various PRT locations.

\section{ACKNOWLEDGMENT}

The work done in this paper is supported by ACCENT5 project of French National Research Agency (ANR).

\section{REFERENCES}

[1] B. F. Boroujeny, "OFDM Versus Filter Bank Multi-carrier, IEEE Signal Processing Magazine," IEEE Signal Processing Magazine, vol. 8, no. 3, pp. 92-112, May 2006.

[2] M. Bellanger, "Specification and design of prototype filter for filter bank based multi-carrier transmission," IEEE International Conference on Acoustic, Speech and Signal Processing, pp. 2417-2420, May 2001.

[3] P. Siohan, C. Siclet, and N. Lacaille, "Analysis and design of OFDM/OQAM systems based on filter bank theory," IEEE Trans. on Signal Processing, vol. 50, pp. 1170-1183, May 2002.

[4] J. Tellado, and J. Cioffi, "Peak power reduction for multicarrier transmission," IEEE CTMC, GLOBECOM, Sydney, Australia, Nov. 1998.

[5] S. Lu, D. Qu, and Y. He, "Sliding Window Tone Reservation Technique for the Peak-to-Average Power Ratio Reduction of FBMC-OQAM Signals," IEEE Wireless Communication Letters, vol. 1, no. 4, pp. 268271, Aug. 2012.

[6] J. Tellado, "Peak to Average Ratio Reduction for Multi-carrier Modulation," Ph.D. Thesis, Stanford University, Stanford, CA, USA, 1999.

[7] R. W. Chang, "Synthesis of band-limited orthogonal signals for multichannel data transmission," Bell System Technical Journal, vol. 45, pp. 1775-1796, Dec. 1966.

[8] B. L. Floch, M. Alard, and C. Berrou, "Coded orthogonal frequency division multiplex," IEEE Proc., vol. 83, no. 6, pp. 982-996, Jun. 1995.

[9] A. Gatherer, and M. Polley, "Controlling clipping in DMT transmission," Conference Record of the Thirsty First Asilomar Conference on Signals, Systems and Computers, vol. 1, pp. 578-584, Nov. 1997.

[10] B. Le Floch, M. Alard, and C. Berrou, "Coded Orthogonal Frequency Division Multiplex, Proceedings of the IEEE, vol. 83, no. 6, pp. 982 996, June 1995. 